\begin{tabular}{|r|l|}
\hline \multicolumn{2}{|c|}{ Statistica Sinica Preprint No: SS-2020-0243 } \\
\hline Title & $\begin{array}{l}\text { Robust Recommendation via Social Network Enhanced } \\
\text { Matrix Completion }\end{array}$ \\
\hline Manuscript ID & SS-2020-0243 \\
\hline URL & http://www.stat.sinica.edu.tw/statistica/ \\
\hline DOI & $10.5705 /$ ss.202020.0243 \\
\hline Complete List of Authors & $\begin{array}{l}\text { Jingxuan Wang, } \\
\text { Haipeng Shen and } \\
\\
\text { Fei Jiang }\end{array}$ \\
\hline Corresponding Author & Fei Jiang \\
\hline E-mail & fei.jiang@ucsf.edu \\
\hline Notice: Accepted version subject to English editing. \\
\hline
\end{tabular}


Statistica Sinica

\title{
ROBUST RECOMMENDATION VIA SOCIAL NETWORK ENHANCED MATRIX COMPLETION
}

\author{
Jingxuan Wang ${ }^{1}$, Haipeng Shen ${ }^{2}$ and Fei Jiang ${ }^{1}$ \\ ${ }^{1}$ University of California, San Francisco and ${ }^{2}$ University of Hong Kong
}

Abstract: Robust product recommendation is crucial for internet platforms to boost their businesses. One challenge though is that the user-product rating matrix often has many missing entries. Social network information generates new insights about user behaviors. To fully utilize the social network information, we develop a novel approach, namely MCNet, which combines the random dot product graph model and the low-rank matrix completion to recover the missing entries in the user-product rating matrix from the internet platform. Our algorithm improves the accuracy and the efficiency of recovering the incomplete matrices. We study the asymptotic properties of the estimator. Furthermore, we perform extensive simulations and show that MCNet outperforms the existing approaches, especially when data have small signals. Moreover, MCNet yields robust estimation under misspecified models. We apply MCNet and the competitors to predict the missing entries in the user-product rating matrices on the Yelp and Douban movie platforms. MCNet generally gives the smallest testing errors among all the comparative methods.

Key words and phrases: Low-rank estimation, matrix completion, missing data, 
random dot product graph, social network.

\section{Introduction}

Uncovering the true user ratings on products is critical for internet platforms, such as Yelp, Facebook, Amazon, to promote their business, because the platforms can utilize the estimated rating to recommend a specific product to the users who may have the highest willingness to pay and in turn maximize the revenue. Such datasets are often arranged in a matrix form where the rows and columns correspond to users and products, respectively. The entries of the matrix are largely missing due to the fact that not every product has been exposed to all users, while the ratings at these missing entries are crucial for establishing the recommendation strategies of the products on the platforms.

Many matrix completion algorithms have been developed to recover the missing entries in the user-product rating matrix, which often adopt certain low-rank estimation frameworks. Srebro, Rennie and Jaakkola (2005) developed a matrix factorization algorithm by assuming the underlying rating matrix has a finite known rank and the missing is completely at random. Recht, Fazel and Parrilo (2010) cast the matrix completion as a constrained convex optimization problem and recovered the matrix through minimizing 
its nuclear norm. Similar convex relaxation has been adopted by Candes and Plan (2010) and Koltchinskii, Lounici and Tsybakov (2011) under different noise settings, while a state-of-art imputation method, namely SoftImpute, was developed to accommodate matrices with growing ranks. Recently, Negahban and Wainwright (2011), Koltchinskii, Lounici and Tsybakov (2011), and Fan, Wang and Zhu (2017) have rigorously studied the statistical properties of the low-rank matrix estimation under the linear regression setting. Klopp (2014) and Elsener and van de Geer (2018) considered using the Huber loss for the robust estimation of the low-rank matrix. Fan, Gong and Zhu (2019) further extended low-rank matrix estimation under the non-linear model framework. In addition, a non-convex relaxation method, namely TopN, was proposed by Kang, Peng and Cheng (2016). Furthermore, deep learning methods have been proposed to recover the missing matrix entries in the recommendation system literature (Liu and Wu (2017); Zhang et al. (2019)). Particularly, Wang, Wang and Yeung (2015) developed a deep learning framework incorporating content information to address the matrix completion problem.

Besides the user-product rating matrix, auxiliary covariates, such as users' demographics, products' attributes, are often collected on the internet platforms. These covariates provide additional information that is not 
explained by the ratings matrix itself (Feuerverger, He and Khatri (2012)). Incorporating the information has been shown to improve both the accuracy and precision of the estimation (Abernethy et al. (2009); Shi, Larson and Hanjalic (2014)). Chiang, Hsieh and Dhillon (2015) and Xu, Jin and Zhou (2013) illustrated the theoretical guarantees on matrix recovery with covariate information under noiseless settings. Furthermore, Mao, Chen and Wong (2019) showed that considering the users' features reduces the matrix estimation error under missing at random settings, where the probability of observation is independent of the unobserved target matrix given the covariates $($ Rubin $(1976))$. Zhu, Shen and Ye (2016) proposed a partial latent model which allows the combined use of both user and item covariates in a linear regression setting with $l_{1}$ and $l_{2}$ penalties. Moreover, by allowing high-dimensional features, Robin et al. (2018) introduced a sparse low-rank estimation to recover the rating matrix and the feature effects simultaneously.

Besides the explicit features collected on the platforms, the social network contains rich information about the associations among the users. The network implicitly tells how much information can be taken from the other users. This information has been translated into the penalty terms in the matrix completion (Ma et al. (2011); Yu, Pan and Li (2011); Liu and Aberer 
(2013)) to reflect the intuition that the closer the two users are, the more information must be taken from each other to recover their missing ratings. Based on the network information, Rao et al. (2015) developed a graph Laplacian method utilizing the network information to assist the matrix completion. Jing et al. (2019) introduced a penalized collaborative filtering method, namely NetRec, allowing users to share the information with their connections in the network. In addition, Dai et al. $(2019)$ developed a smooth recommendation system based on the latent factor model which jointly incorporates social network, product network, and user-product specific covariates through kernel weighting.

When the covariate information is not given, the missingness of the matrix entries is considered to be missing completely at random (Srebro, Rennie and Jaakkola (2005); Candes and Plan (2010); Koltchinskii, Lounici and Tsybakov (2011)). This assumption is not appropriate in our setting as different users may have significantly different willingness for rating. The additional network information will generate user specific features, which allows us to estimate the missing probability tailored to the individual features. To estimate the user specific missing probability, Mao, Wong and Chen (2018) introduced a two-step inverse probability weighting based matrix completion framework where the observation probabilities are esti- 
mated via a generalized linear model with low-rank predictor matrix. Furthermore, Bi et al. (2017) proposed a singular value decomposition based group-specific model to utilize the between-subject dependency information from users and items which share similar missingness characteristics.

In this article, we propose a matrix completion social network (MCNet) algorithm which utilizes the social network information to improve the matrix completion. Specifically, we embed the high-dimensional network structure into the low-dimensional space through the adjacency spectral embedding (Sussman et al. (2012); Lyzinski et al. (2014); Athreya et al. (2018)) and generate a set of latent positions which best summarizes the distances among the users. We then incorporate this embedding into the matrix completion model to improve the accuracy of recovering missing entries in the rating matrix. The theoretical guarantee of the adjacency spectral embedding has been studied by Oliveira (2009), Lu and Peng (2013), and Lei and Rinaldo (2015). Formally, Sussman, Tang and Priebe (2013) and Lyzinski et al. (2016) established the estimation consistency, while Athreya et al. (2016) and Tang and Priebe 2018) provided asymptotic normality of the resulting latent positions.

The contributions of the paper are five-fold. (1) We develop a flexible model incorporating the network information to improve the accuracy of 
matrix recovery. (2) We provide an efficient estimation procedure, which yields smaller estimation errors comparing with the competing methods proposed by Mazumder, Hastie and Tibshirani (2010), Kang, Peng and Cheng (2016), and Jing et al. (2019). (3) Our method is flexible to consider different missing mechanisms, including covariate-independent and covariate-dependent missingness. (4) We provide asymptotic upper bounds of the estimation errors. (5) We show that our algorithm improves both the accuracy and the efficiency of recovering the incomplete matrices in the simulation studies and real data analysis.

The rest of the article is organized as follows. In Section 2, we describe the MCNet model and the estimation procedure. In Section 3, we provide the theoretic results. We evaluate the MCNet method and compare it with the existing methods through extensive simulations in Section 4. We apply MCNet to analyze data from Douban Movie and Yelp Dataset Challenge in Section 5 and provide conclusive remarks in Section 6.

\section{Methodology}

\section{$2.1 \quad$ Notation}

Before presenting the model, we define the notations that we will use throughout the article. For a $n_{1} \times n_{2}$ matrix $\mathbf{H}$, we denote $H_{i k}$ as the 
$i, k$ th entry, $\mathbf{H}_{i}$. as the $i$ th row, $\mathbf{H}_{\cdot k}$ as the $k$-th column. We define $\lambda_{i}(\mathbf{H})$ to be the $i$ th largest eigenvalue of $\mathbf{H}$, and $\sigma_{i}(\mathbf{H})=\sqrt{\lambda_{i}\left(\mathbf{H}^{\mathrm{T}} \mathbf{H}\right)}$ to be the $i$ th largest singular value of $\mathbf{H}$. Besides, $\|\mathbf{H}\|_{2},\|\mathbf{H}\|_{\max },\|\mathbf{H}\|_{F}$, and $\|\mathbf{H}\|_{*}$ denote the operator norm, element-wise maximum norm, Frobenius norm, and nuclear norm of the matrix $\mathbf{H}$, respectively. Furthermore, we denote $\|\mathbf{H}\|_{2 \rightarrow \infty}$ as the maximum of Euclidean norms for rows of $\mathbf{H}$, that is, $\|\mathbf{H}\|_{2 \rightarrow \infty}=\max _{i}\left\{\left\|\mathbf{H}_{i}^{\mathrm{T}}\right\|_{2}\right\} . \quad\left\langle\mathbf{H}_{1}, \mathbf{H}_{2}\right\rangle$ denotes the trace inner product between $\mathbf{H}_{1}$ and $\mathbf{H}_{2}$. Finally, we define the signal in the matrix $\mathbf{H}$ as $\sum_{i j}\left\{H_{i j}-\sum_{i j} H_{i j} /\left(n_{1} n_{2}\right)\right\}^{2} /\left(n_{1} n_{2}-1\right)$.

\subsection{The MCNet Model}

Let $M_{i k}$ be the connection indicator such that $M_{i k}=M_{k i}=1$ if the $i$ th and the $k$ th users are connected with each other in the social network. Furthermore, we define $\mathbf{M}$ as the adjacency matrix with $M_{i k}$ being its $i, k$ th entry, and assume

$$
\operatorname{Pr}(\mathbf{M} \mid \mathbf{X})=\prod_{i<k}\left(\mathbf{X}_{i} \cdot \mathbf{X}_{k}^{\mathrm{T}}\right)^{M_{i k}}\left(1-\mathbf{X}_{i} \cdot \mathbf{X}_{k}^{\mathrm{T}}\right)^{1-M_{i k}}
$$

where $\mathbf{X}_{i} . \in \mathbb{R}^{1 \times d}$ and $\mathbf{X}=\left(\mathbf{X}_{1}^{\mathrm{T}}, \ldots, \mathbf{X}_{n_{1}}^{\mathrm{T}} .\right)^{\mathrm{T}} \in \mathbb{R}^{n_{1} \times d}$ is the latent position matrix. We say that $\mathbf{M}$ follows a random dot product graph (RDPG) distribution with the latent position $\mathbf{X}$, denoted by $\mathbf{M} \sim \operatorname{RDPG}(\mathbf{X})$.

In addition, let $Y_{i k}$ be the rating for the product $k$ from the user $i$, and 
we assume

$$
Y_{i k}=A_{0 i k}+\epsilon_{i k}
$$

where $\left\{\epsilon_{i k}, i=1, \ldots, n_{1}, k=1, \ldots, n_{2}\right\}$ are independent mean zero random errors. We assume that $\mathbf{Y}$ and $\mathbf{M}$ are independent when $\mathbf{X}$ is given. Let $\mathbf{A}_{0}$ be the matrix with the $i, k$ th entry being $A_{0 i k}$, and we further decompose the information in $\mathbf{A}_{0}$ as

$$
\mathbf{A}_{0}=\mathbf{X} \boldsymbol{\beta}_{0}+\mathbf{B}_{0}
$$

Here $\boldsymbol{\beta}_{0} \in \mathbb{R}^{d \times n_{2}}$ is the unknown parameter matrix of interest, and $\mathbf{B}_{0} \in$ $\mathbb{R}^{n_{1} \times n_{2}}$ is an unknown low-rank matrix whose columns are orthogonal to the column space of $\mathbf{X}$ so that $\mathbf{P}_{\mathbf{X}} \mathbf{B}_{0}=\mathbf{0}$, where $\mathbf{P}_{\mathbf{X}}=\mathbf{X}\left(\mathbf{X}^{\mathrm{T}} \mathbf{X}\right)^{-1} \mathbf{X}^{\mathrm{T}}$. The low-rank assumption is commonly used in the matrix factorization literature (Srebro, Rennie and Jaakkola (2005); Recht, Fazel and Parrilo (2010); Candes and Plan (2010); Koltchinskii, Lounici and Tsybakov (2011)), which assumes there are a few latent factors explaining most of the data. This assumption allows information borrowed across all observed entries in $\mathbf{Y}$. Furthermore, we assume $\mathbf{B}_{0}$ is orthogonal to the column space of $\mathbf{X}$ to ensure the model is identifiable. To see that, suppose $\mathbf{P}_{\mathbf{X}} \mathbf{B} \neq \mathbf{0}$, it is easy to see that $\mathbf{X} \boldsymbol{\beta}_{0}+\mathbf{B}_{0}=\mathbf{X}\left\{\boldsymbol{\beta}_{0}+\left(\mathbf{X}^{\mathrm{T}} \mathbf{X}\right)^{-1} \mathbf{X}^{\mathrm{T}} \mathbf{B}_{0}\right\}+\mathbf{P}_{\mathbf{X}}^{\perp} \mathbf{B}_{0}$, where $\mathbf{P}_{\mathbf{X}}^{\perp}=\mathbf{I}-\mathbf{X}\left(\mathbf{X}^{\mathrm{T}} \mathbf{X}\right)^{-1} \mathbf{X}^{\mathrm{T}}$. Now $\left\{\boldsymbol{\beta}_{0}+\left(\mathbf{X}^{\mathrm{T}} \mathbf{X}\right)^{-1} \mathbf{X}^{\mathrm{T}} \mathbf{B}_{0}\right\}$ is an unknown pa- 
rameter since $\boldsymbol{\beta}_{0}, \mathbf{B}_{0}$ are both unknown. Furthermore, $\mathbf{P}_{\mathbf{X}}^{\perp} \mathbf{B}_{0}$ is orthogonal to $\mathbf{X}$. Therefore, for any $\mathbf{B}_{0}, \mathbf{X} \boldsymbol{\beta}_{0}+\mathbf{B}_{0}$ can be decomposed to a linear combination of $\mathbf{X}$ and a matrix orthogonal to the column space of $\mathbf{X}$. This assumption is also used in Mao, Chen and Wong (2019).

\subsection{MCNet Estimation}

When $\mathbf{Y}$ and $\mathbf{X}$ are fully observed, $\boldsymbol{\beta}_{0}$ and $\mathbf{B}_{0}$ are the minimizers of

$$
E\|\mathbf{Y}-\mathbf{X} \boldsymbol{\beta}-\mathbf{B}\|_{F}^{2}
$$

Because each user only rates a subset of the products, $\mathbf{Y}$ contains missing entries. Let $W_{i k}=1$ if the product $k$ has a rating from user $i$, and $\theta_{i k}$ be the true probability of the $i, k$ th entry being observed, let $\mathbf{W}, \boldsymbol{\Omega}_{0}$ be the matrices with the $i, k$ th entry being $W_{i k}$ and $\theta_{i k}^{-1}$ respectively. Then we consider the risk function

$$
L(\boldsymbol{\beta}, \mathbf{B})=E\left\|\mathbf{Y} \circ \mathbf{W} \circ \boldsymbol{\Omega}_{0}-\mathbf{X} \boldsymbol{\beta}-\mathbf{B}\right\|_{F}^{2},
$$

where $\circ$ is the Hadamard product. Under Conditions (C1) and (C3) in the next section, $\boldsymbol{\beta}_{0}$ and $\mathbf{B}_{0}$ are the minimizers of $L(\boldsymbol{\beta}, \mathbf{B})$ because $E(\mathbf{Y} \circ \mathbf{W} \circ$ $\left.\boldsymbol{\Omega}_{0} \mid \mathbf{X}\right)=\mathbf{X}^{\mathrm{T}} \boldsymbol{\beta}_{0}+\mathbf{B}_{0}$ and the partial derivative of $E\left\|\mathbf{Y} \circ \mathbf{W} \circ \boldsymbol{\Omega}_{0}-\mathbf{X} \boldsymbol{\beta}-\mathbf{B}\right\|_{F}^{2}$ with respect to $\boldsymbol{\beta}$ and $\mathbf{B}$ are zeros when $\boldsymbol{\beta}=\boldsymbol{\beta}_{0}$ and $\mathbf{B}=\mathbf{B}_{0}$. In addition, 
since $\mathbf{X}$ and $\mathbf{B}_{0}$ are orthogonal, it is easy to see that

$$
\begin{aligned}
L\left(\boldsymbol{\beta}_{0}, \mathbf{B}_{0}\right)= & E\left\|\mathbf{X} \boldsymbol{\beta}_{0}-\mathbf{P}_{\mathbf{X}}\left(\mathbf{W} \circ \boldsymbol{\Omega}_{0} \circ \mathbf{Y}\right)\right\|_{F}^{2} \\
& +E\left\|\mathbf{B}_{0}-\mathbf{P}_{\mathbf{X}}^{\perp}\left(\mathbf{W} \circ \boldsymbol{\Omega}_{0} \circ \mathbf{Y}\right)\right\|_{F}^{2} .
\end{aligned}
$$

In the above equation, $\boldsymbol{\beta}_{0}$ and $\mathbf{B}_{0}$ are in two separate loss functions, which allows us to estimate $\boldsymbol{\beta}_{0}$ and $\mathbf{B}_{0}$ separately.

However, $\mathbf{X}$ is generally unobservable. We estimate it by using the relation in 2.1 as follows. Because $\mathbf{M}$ is a square matrix, we can write $\mathbf{M}=\sum_{i=1}^{n_{1}} \lambda_{i}(\mathbf{M}) \mathbf{u}_{i} \mathbf{u}_{i}^{\mathrm{T}}$ where the $\lambda_{i}(\mathbf{M})$ 's are the eigenvalues ordered by absolute magnitudes and $\mathbf{u}_{1}, \ldots, \mathbf{u}_{n_{1}}$ are the corresponding eigenvectors. Let $\mathbf{U}_{\mathbf{M}}=\left(\mathbf{u}_{1}, \ldots, \mathbf{u}_{d}\right)$ and $\mathbf{S}_{\mathbf{M}}=\operatorname{diag}\left\{\left|\lambda_{1}(\mathbf{M})\right|, \ldots,\left|\lambda_{d}(\mathbf{M})\right|\right\}$, where the hyperparameter $d$ can be prescribed by the eigenvalue ratio test Ahn and Horenstein (2013)). That is,

$$
d=\underset{q}{\operatorname{argmax}} \frac{\log \left(V_{q-1} / V_{q}\right)}{\log \left(V_{q} / V_{q+1}\right)},
$$

where $V_{q}$ is the sum of the first $q$ largest absolute eigenvalues of $\mathbf{M}$. We then obtain the estimator $\widehat{\mathbf{X}}=\mathbf{U}_{\mathbf{M}} \mathbf{S}_{\mathbf{M}}^{1 / 2}$ through the adjacency spectral embedding that gives a continuous representation of nodes in social network as vectors in a low-dimensional space.

We replace $\mathbf{X}$ in $L(\boldsymbol{\beta}, \mathbf{B})$ by the adjacency spectral embedding $\widehat{\mathbf{X}}$, and 
obtain the estimators as

$$
\widehat{\boldsymbol{\beta}}=\underset{\boldsymbol{\beta} \in \mathbb{R}^{d \times n_{2}}}{\operatorname{argmin}}\left\{\frac{1}{n_{1} n_{2}}\left\|\widehat{\mathbf{X}} \boldsymbol{\beta}-\mathbf{P}_{\widehat{\mathbf{X}}}(\mathbf{W} \circ \widehat{\boldsymbol{\Omega}} \circ \mathbf{Y})\right\|_{F}^{2}+\lambda_{1}\|\boldsymbol{\beta}\|_{F}^{2}\right\},
$$

and

$$
\begin{aligned}
\widehat{\mathbf{B}}= & \underset{\mathbf{B} \in \mathcal{N}(\widehat{\mathbf{X}})}{\operatorname{argmin}}\left\{\frac{1}{n_{1} n_{2}}\left\|\mathbf{B}-\mathbf{P}_{\widehat{\mathbf{X}}}^{\perp}(\mathbf{W} \circ \widehat{\mathbf{\Omega}} \circ \mathbf{Y})\right\|_{F}^{2}\right. \\
& \left.+\lambda_{2}\left\{\alpha\|\mathbf{B}\|_{*}+(1-\alpha)\|\mathbf{B}\|_{F}^{2}\right\}\right\}
\end{aligned}
$$

where $\mathcal{N}(\widehat{\mathbf{X}})$ is the space orthogonal to the one spanned by $\widehat{\mathbf{X}}$, and $\widehat{\Omega}$ is an element-wise consistent estimator for $\boldsymbol{\Omega}_{0}$. In 2.4 , the term $\|\boldsymbol{\beta}\|_{F}^{2}$ leads to a ridge regression problem. In 2.5 , the nuclear term $\|\mathbf{B}\|_{*}$ has a thresholding effect over singular values because it's equivalent to the $l_{1}$ norm penalty of the vector of singular values. The Frobenius term $\|\mathbf{B}\|_{F}^{2}=\operatorname{trace}\left(\mathbf{B}^{\mathrm{T}} \mathbf{B}\right)=$ $\sum_{i}^{n_{1} \wedge n_{2}} \sigma_{i}^{2}$ corresponds to the $l_{2}$ norm penalty of the singular values. The convex regularization leads to elastic-net regularization of singular values (Zou and Hastie (2005); Sun and Zhang (2012)). The parameter $\alpha$ controls the balance of the two penalties. The strong convex Frobenius penalty is introduced to improve finite sample performance (Sun and Zhang (2012); Mao, Chen and Wong (2019)) and stability under highly corrupted data (Li, Chen and Li (2012); Kim, Lee and Oh (2015)).

The estimation in 2.4 leads to

$$
\widehat{\boldsymbol{\beta}}=\left(\widehat{\mathbf{X}}^{\mathrm{T}} \widehat{\mathbf{X}}+n_{1} n_{2} \lambda_{1} \mathbf{I}\right)^{-1} \widehat{\mathbf{X}}^{\mathrm{T}}(\mathbf{W} \circ \widehat{\boldsymbol{\Omega}} \circ \mathbf{Y}) .
$$


From 2.5$), \widehat{\mathbf{B}}$ can be obtained using the one-step first-order scaled singularvalue-thresholding operator based on the algorithm introduced in Cai, Candes and Shen (2010). We show that $\widehat{\mathbf{B}}$ is guaranteed to be orthogonal to the column space of $\widehat{\mathbf{X}}$ in Section $\mathrm{S} 1.2$ of the supplementary material. Now because $\widehat{\mathbf{X}}$ converges to $\mathbf{X}$ in Frobenius norm asymptotically (shown in the next section), $\widehat{\mathbf{B}}$ is orthogonal to the column space of $\mathbf{X}$ asymptotically. Finally, we estimate $\mathbf{A}_{0}$ by $\widehat{\mathbf{A}}=\widehat{\mathbf{X}} \widehat{\boldsymbol{\beta}}+\widehat{\mathbf{B}}$. We illustrate the estimation procedure in Algorithm 1 .

Algorithm 1 MCNet algorithm.

1. Estimate $\mathbf{X}$ by adjacency spectral embedding $\widehat{\mathbf{X}}=\mathbf{U}_{\mathbf{M}} \mathbf{S}_{\mathbf{M}}^{1 / 2}$

2. Estimate $\boldsymbol{\beta}_{0}$ by $\widehat{\boldsymbol{\beta}}=\left(\widehat{\mathbf{X}}^{\mathrm{T}} \widehat{\mathbf{X}}+n_{1} n_{2} \lambda_{1} \mathbf{I}\right)^{-1} \widehat{\mathbf{X}}^{\mathrm{T}}(\mathbf{W} \circ \widehat{\boldsymbol{\Omega}} \circ \mathbf{Y})$

3. Estimate $\mathbf{B}_{0}$ by $\widehat{\mathbf{B}}=\mathbf{U D}_{n_{1}, n_{2}, \lambda_{2}, \alpha} \mathbf{V}^{\mathrm{T}}$ where $\mathbf{U D V}^{\mathrm{T}}$ is the singular value decomposition of $\mathbf{P}_{\widehat{\mathbf{X}}}^{\perp}(\mathbf{W} \circ \widehat{\boldsymbol{\Omega}} \circ \mathbf{Y}), \quad \mathbf{D}_{n_{1}, n_{2}, \lambda_{2}, \alpha}=$ $\operatorname{diag}\left\{\frac{\left(D_{1,1}-n_{1} n_{2} \lambda_{2} \alpha / 2\right)_{+}}{1+n_{1} n_{2} \lambda_{2}(1-\alpha)}, \ldots, \frac{\left(D_{n_{1} \wedge n_{2}, n_{1} \wedge n_{2}}-n_{1} n_{2} \lambda_{2} \alpha / 2\right)_{+}}{1+n_{1} n_{2} \lambda_{2}(1-\alpha)}\right\}$, and $t_{+}=t \vee 0$

4. Return $\widehat{\mathbf{A}}=\widehat{\mathbf{X}} \widehat{\boldsymbol{\beta}}+\widehat{\mathbf{B}}$

\section{Main results}

We define $d\left(\mathbf{H}_{1}, \mathbf{H}_{2}\right)=\left\|\mathbf{H}_{1}-\mathbf{H}_{2}\right\|_{F} / \sqrt{d_{1} d_{2}}$ for $d_{1} \times d_{2}$ matrices $\mathbf{H}_{1}, \mathbf{H}_{2}$. Because $\mathbf{X X}^{\mathrm{T}}=\mathbf{X O O}^{\mathrm{T}} \mathbf{X}^{\mathrm{T}}$ for an orthogonal matrix $\mathbf{O}, \mathbf{X}$ and in turn $\boldsymbol{\beta}$ are only identifiable up to an orthogonal transformation. We show the convergences of $\widehat{\boldsymbol{\beta}}$ to $\mathbf{O}^{\mathrm{T}} \boldsymbol{\beta}_{0}, \widehat{\mathbf{B}}$ to $\mathbf{B}_{0}$, and that of $\widehat{\mathbf{A}}$ to $\mathbf{A}_{0}$ by establishing 
the upper bounds of $\left\|\widehat{\boldsymbol{\beta}}_{\cdot j}-\mathbf{O}^{\mathrm{T}} \boldsymbol{\beta}_{0, \cdot j}\right\|_{2}, d\left(\widehat{\mathbf{B}}, \mathbf{B}_{0}\right)$, and $d\left(\widehat{\mathbf{A}}, \mathbf{A}_{0}\right)$, respectively. Before that, we present the technical conditions needed for our analysis.

(C1) For all $i, j, \epsilon_{i j}$ is independent random error with zero mean. There exist positive constants $c_{\sigma}$ and $\eta$ such that $\max _{i, j} E\left|\epsilon_{i j}\right|^{l} \leq l ! c_{\sigma}^{2} \eta^{l-2} / 2$ holds for any integer $l \geq 2$.

(C2) There exist positive constants $a_{1}, a_{2}$ and $a_{3}$ such that

$$
\begin{aligned}
\left\|\mathbf{A}_{0}\right\|_{\max } & \leq \sqrt{\log \left(n_{1}+n_{2}\right)} a_{1} \\
\left\|\mathbf{A}_{0}\right\|_{2 \rightarrow \infty} & \leq a_{2} \sqrt{n_{2}} \\
\left\|\mathbf{A}_{0}^{\mathrm{T}}\right\|_{2 \rightarrow \infty} & \leq a_{2} \sqrt{n_{1}} \\
\left\|\boldsymbol{\beta}_{0}\right\|_{\max } & \leq a_{3}
\end{aligned}
$$

(C3) For all $i, j$, the observation indicator $W_{i j}$ independently follows the Bernoulli distribution with parameter $\theta_{i j}:=\operatorname{Pr}\left(W_{i j}=1 \mid \mathbf{X}_{i, \cdot}, Y_{i j}\right)=$ $\operatorname{Pr}\left(W_{i j}=1 \mid \mathbf{X}_{i, .}\right) \in(0,1)$. In addition, $W_{i j}$ is independent of $\epsilon_{i j}$.

(C4) (a) There exists a lower bound $\theta_{L} \in(0,1)$ such that $\theta_{i j} \geq \theta_{L}$ for all $i, j$. $\theta_{L}$ can vanish when $n_{1}, n_{2} \rightarrow \infty$. (b) For all $i, j,\left|\widehat{\theta}_{i j}-\theta_{i j}\right|=O_{p}\left(n_{1}^{-1 / 2}\right)$ and $\widehat{\theta}_{i j}$ is independent of $\left\{\epsilon_{i j}\right\}$.

(C5) (a) The true latent position matrix $\mathbf{X}$ has rank $d$. (b) $n_{1}^{-1} \mathbf{X}^{\mathrm{T}} \mathbf{X} \rightarrow \mathbf{S}_{x}$ as $n_{1} \rightarrow \infty,\left\|\mathbf{S}_{x}\right\|_{2}<\infty$ and $\lambda_{d}\left(\mathbf{S}_{x}\right) \geq c_{0}$ where $c_{0}$ is a positive con- 
stant. (c) $\max _{i \leq n_{1}} \sum_{j=1}^{n_{1}} \Pi_{i j}>\log ^{4+a}\left(n_{1}\right)$ for some positive constant $a$, where $\boldsymbol{\Pi}=\mathbf{X X}^{\mathrm{T}}$.

Conditions (C1), (C4) are standard conditions discussed in Mao, Chen and Wong (2019) to achieve estimation consistency when incorporating covariate information in matrix completion. Condition (C3) assumes that the missingness follows either covariate-independent or covariate-dependent missingness mechanisms. Under covariate-independent settings (Koltchinskii, Lounici and Tsybakov (2011); Recht (2011)), we estimate the observed probability by $\sum_{i j} W_{i j} /\left(n_{1} n_{2}\right)$. Under the covariate-dependent missingness, if $\theta_{L}$ in Condition (C4) is bounded from below, we can use the standard logistic regression

$$
\operatorname{logit}\left(\theta_{i j}\right)=\tau_{0 j}+\mathbf{X}_{i} \cdot \boldsymbol{\tau}_{j}
$$

to generate root- $n$ consistent estimators for the observed probabilities. If $\theta_{L}$ vanishes as $n_{1}, n_{2}$ go to infinity, we can use a zero-inflated binomial regression to generate root- $n$ consistent estimators (Diallo, Diop and Dupuy (2017); Hall (2000)). Condition (C5) is a global convex condition, which guarantees the consistency of recovering the latent position in the random dot product model. 
Lemma 3.1. Assume Condition (C5) holds, there is an orthogonal matrix $\mathbf{O} \in \mathbb{R}^{d \times d}$ such that $\left\|\widehat{\mathbf{X}} \mathbf{O}^{\mathrm{T}}-\mathbf{X}\right\|_{F}=O_{p}\left(n_{1}^{1 / 4}\right)$.

In Lemma 3.1, we employ the Hoeffding Concentration Theorem and assume the probability of $M_{i j}=1$ is strictly greater than 0 for all $i, j$. The lemma is based on Lemma S1.3 in the supplementary material, which is equivalent to Lemma 50 in Athreya et al. (2018) and Theorem A.5 in Tang et al. (2017) under Condition (C5) that the eigenvalues of the probability matrix $\Pi$ grow linearly with $n_{1}$. This bound allows us to achieve the root- $n$ convergence of $\widehat{\boldsymbol{\beta}}$ to the truth.

Theorem 3.1. Assume that Conditions (C1) (C5) hold and $\lambda_{1}=o\left(n_{2}^{-1}\right)$. Then there exist an orthogonal matrix $\mathbf{O}$ and constant $c$ such that $\| \widehat{\boldsymbol{\beta}}_{\cdot j}-$ $\mathbf{O}^{\mathrm{T}} \boldsymbol{\beta}_{0, j} \|_{2}=O_{p}\left[\max \left\{n_{1}^{-1 / 2} \log ^{c}\left(n_{1}\right) \theta_{L}^{-1}, n_{1}^{-1} \delta^{1 / 2}(\boldsymbol{\Pi})\right\}\right]$ for each $j=1, \ldots, n_{2}$.

Theorem 3.1 gives the parametric convergence rate for $\widehat{\boldsymbol{\beta}}_{\cdot j}$ to the truth up to an orthogonal transformation. The fact that $\widehat{\mathbf{X}}$ instead of the true $\mathbf{X}$ was used in the loss function does not inflate the asymptotic errors. And it is easy to see that $d\left(\widehat{\boldsymbol{\beta}}, \mathbf{O}^{\mathrm{T}} \boldsymbol{\beta}_{0}\right)=O_{p}\left[\max \left\{n_{1}^{-1 / 2} \log ^{c}\left(n_{1}\right) \theta_{L}^{-1}, n_{1}^{-1} \delta^{1 / 2}(\boldsymbol{\Pi})\right\}\right]$ because $\widehat{\boldsymbol{\beta}}-\boldsymbol{\beta}_{0}$ has a finite rank. The proof is provided in the supplement.

Lemma 3.2. Assume Conditions (C1) (C5) hold, and $\lambda_{1}=o\left(n_{2}^{-1}\right)$, then

$$
d^{2}\left(\widehat{\mathbf{X}} \widehat{\boldsymbol{\beta}}, \mathbf{X} \boldsymbol{\beta}_{0}\right)=O_{p}\left\{\max \left(\frac{\log ^{2 c}\left(n_{1}\right) \delta(\boldsymbol{\Pi})}{n_{1}^{2} \theta_{L}^{2}}, \frac{\delta^{2}(\boldsymbol{\Pi})}{n_{1}^{3}}, \frac{\left\|\boldsymbol{\beta}_{0}\right\|_{F}^{2}}{n_{1}^{1 / 2} n_{2}}\right)\right\}
$$


Lemma 3.2 is a direct consequence of Lemma 3.1 and Theorem 3.1, and is necessary to establish the convergency of $\widehat{\mathbf{A}}$.

Lemma 3.3. Assume Conditions (C1) (C5) hold, $\alpha \in(0,1], \lambda_{2} \geq\left\{2 c_{Y} \log ^{c}\left(n_{1}\right) \theta_{L}^{-1}+\right.$ $\left.a_{2}\right\} / \sqrt{n_{1} n_{2} \alpha^{2}}+2\left\|\mathbf{B}_{0}\right\|_{2} /\left(n_{1} n_{2} \alpha\right)$ for any positive constant $c_{Y}$, then

$$
d^{2}\left(\widehat{\mathbf{B}}, \mathbf{B}_{0}\right)=O_{p}\left[\max \left\{\lambda_{2} \alpha\left\|\mathbf{B}_{0}\right\|_{*}, \lambda_{2}(1-\alpha)\left\|\mathbf{B}_{0}\right\|_{F}^{2}\right\}\right]
$$

Lemma 3.3, together with Lemma 3.2, leads to

Theorem 3.2. Assume Conditions (C1) (C5) hold and $\lambda_{1}=o\left(n_{2}^{-1}\right)$. Fur-

thermore, for any given $c_{Y}>0$, we assume $\lambda_{2} \geq\left\{2 c_{Y} \log ^{c}\left(n_{1}\right) \theta_{L}^{-1}+a_{2}\right\} / \sqrt{n_{1} n_{2} \alpha^{2}}+$ $2\left\|\mathbf{B}_{0}\right\|_{2} /\left(n_{1} n_{2} \alpha\right)$. Then

$d^{2}\left(\widehat{\mathbf{A}}, \mathbf{A}_{0}\right)=O_{p}\left[\max \left\{\frac{\log ^{2 c}\left(n_{1}\right) \delta(\boldsymbol{\Pi})}{n_{1}^{2} \theta_{L}^{2}}, \frac{\delta^{2}(\boldsymbol{\Pi})}{n_{1}^{3}}, \frac{\left\|\boldsymbol{\beta}_{0}\right\|_{F}^{2}}{n_{1}^{1 / 2} n_{2}}, \lambda_{2} \alpha\left\|\mathbf{B}_{0}\right\|_{*}, \lambda_{2}(1-\alpha)\left\|\mathbf{B}_{0}\right\|_{F}^{2}\right\}\right]$.

Remark 1. Comparing with the state-of-the-art SoftImpute algorithm Koltchinskii, Lounici and Tsybakov (2011), $d^{2}\left(\widehat{\mathbf{A}}, \mathbf{A}_{0}\right)$ of MCNet grows with $\left\|\boldsymbol{\beta}_{0}\right\|_{F},\left\|\mathbf{B}_{0}\right\|_{*}$, and $\left\|\mathbf{B}_{0}\right\|_{F}$, while that of SoftImpute increases with $\left\|\mathbf{A}_{0}\right\|_{*}$ and $\left\|\mathbf{A}_{0}\right\|_{F}$ as shown in Corollary 1 of Koltchinskii, Lounici and Tsybakov (2011). In general, $\left\|\mathbf{A}_{0}\right\|_{*} \geq\left\|\mathbf{B}_{0}\right\|_{*}$ and $\left\|\mathbf{A}_{0}\right\|_{F}$ is greater than both $\left\|\boldsymbol{\beta}_{0}\right\|_{F}$ and $\left\|\mathbf{B}_{0}\right\|_{F}$. Hence MCNet improves estimation accuracy by incorporating the social network information.

Comparing with the most recent social network collaborative filtering method NetRec Jing et al. (2019), Theorem 3.2 shows that the estimation 
error of the social network structure, that is $\widehat{\mathbf{X}}$, does not contribute to $d^{2}\left(\widehat{\mathbf{A}}, \mathbf{A}_{0}\right)$. On the other hand, for NetRec, its $d^{2}\left(\widehat{\mathbf{A}}, \mathbf{A}_{0}\right)$ increases with the additional bias induced by the social network penalty as shown in Theorem 3.1 of Jing et al. (2019). This implies that, when the signal in $\mathbf{A}_{0}$ is relatively small compared with this social network bias, NetRec will have poor performance while MCNet will still provide satisfactory results. We demonstrate this point through simulations in Section 4.2 .

\section{Simulations}

We evaluate the convergence of MCNet, and compare it with SoftImpute in Mazumder, Hastie and Tibshirani (2010), the TopN proposed in Kang, Peng and Cheng (2016), and the NetRec method proposed in Jing et al. (2019).

\subsection{Performance as the signal-to-noise ratio varies}

We fix $d=10$ and choose $n_{1}=n_{2}=500$ to 2000 by stepsize 250 . Each entry of $\mathbf{X}$ is generated from a beta distribution with parameters $(1.5,1)$. We then scale each entry by a chosen constant to ensure $\max _{i, j} \mathbf{X}_{i} \cdot \mathbf{X}_{j}^{\mathrm{T}}=$ 0.9. Furthermore, we generate $M_{i j}=M_{j i}, i \neq j$ from a Bernoulli distribution with success rate $\mathbf{X}_{i} \cdot \mathbf{X}_{j}^{\mathrm{T}}$ and generate each entry in $\boldsymbol{\beta}_{0}$ from a 
4.1 Performance as the signal-to-noise ratio varies19

mean zero normal distribution with variance $d / n_{2}$. Moreover, we define $\mathbf{B}_{0}=\mathbf{P}_{\mathbf{X}}^{\perp} \mathbf{U}_{0} \mathbf{V}_{0}^{\mathrm{T}} d / \sqrt{n_{1} n_{2}}$ where $\mathbf{U}_{0} \in \mathbb{R}^{n_{1} \times 10}$ and $\mathbf{V}_{0} \in \mathbb{R}^{n_{2} \times 10}$ are matrices with standard normal entries. Let $\mathbf{A}_{0}=\mathbf{X} \boldsymbol{\beta}_{0}+\mathbf{B}_{0}$ and $\mathbf{Y}=\mathbf{A}_{0}+\boldsymbol{\epsilon}$, where $\boldsymbol{\epsilon}$ is an error matrix with independent mean zero normal entries. The standard deviations of the errors are chosen to achieve $1,0.5,0.2,0.1$, and 0.05 signal-to-noise ratios.

We adopt two types of missing mechanisms. In Model I, we consider covariate-independent missingness with $\theta_{i j}=0.2$ uniformly across all $i, j$ 's. In Model II, the missing probability follows the logistic model in (3.1) where the regression parameters $\tau_{0 j}$ and $\boldsymbol{\tau}_{j}$ are selected to achieve $20 \%$ missing rates in $\mathbf{Y}$ on average.

We select the tuning parameters $\lambda_{1}, \lambda_{2}$, and $\alpha$ by the error perturbation method introduced in Jing et al. (2019) in the simulations. More specifically, given $\mathbf{A}_{0}$, we generate $\boldsymbol{\epsilon}$ through a normal distribution and calculate $d\left(\widehat{\mathbf{A}}, \mathbf{A}_{0}\right)$ after the estimation. We repeat the procedure $K$ times and obtain the average of $d\left(\widehat{\mathbf{A}}, \mathbf{A}_{0}\right)$ as the residual mean square error (RMSE). Then, we select tuning parameters that yield the smallest RMSE on average. The same procedure is adopted for selecting tuning parameters in the SoftImpute, TopN, and NetRec procedures.

We evaluate the convergence of MCNet, and compare the RMSEs from 
4.2 Performance as the noise level varies20

MCNet, SoftImpute, TopN, and NetRec. Figure 1 and Figure 2 show the results when the entries are missing according to Model I and Model II, respectively. They indicate that the convergence rates of $d\left(\widehat{\boldsymbol{\beta}}, \mathbf{O}^{\mathrm{T}} \boldsymbol{\beta}_{0}\right)$, $d\left(\widehat{\mathbf{X}} \mathbf{O}^{\mathrm{T}}, \mathbf{X}\right)$, and $d\left(\widehat{\mathbf{B}}, \mathbf{B}_{0}\right)$ are consistent with the theoretic rates derived in Theorem 3.1, Lemma 3.1, and Lemma 3.3, which are shown as the dashed curves. Here, the specific form of the orthogonal matrix $\mathbf{O}$ is described in the online supplementary material. The results also suggest that MCNet has smaller $d\left(\widehat{\mathbf{A}}, \mathbf{A}_{0}\right)$ compared with those from SoftImpute, TopN, and NetRec. The advantages are more obvious when the signal-to-noise ratio becomes smaller. Moreover, we summarize the square root of the area under the $d^{2}\left(\widehat{\mathbf{A}}, \mathbf{A}_{0}\right)$ curves under different settings in Table 1 , which shows that MCNet outperforms SoftImpute, TopN, and NetRec in all settings with smaller area under the curve on average.

\subsection{Performance as the noise level varies}

We compare MCNet, SoftImpute, TopN, and NetRec under the models with various noise levels $\sigma_{i j}$ and provide guidelines for choosing among the four methods in practice. The parameters $d, \boldsymbol{\beta}_{0}, \mathbf{B}_{0}$ and $\mathbf{X}$ are assumed to be the same as those in Section 4.1. We choose $n_{1}=n_{2}=500$ to 2000 by stepsize 500. We generate the observation matrix through $\mathbf{Y}=$ 
4.2 Performance as the noise level varies21
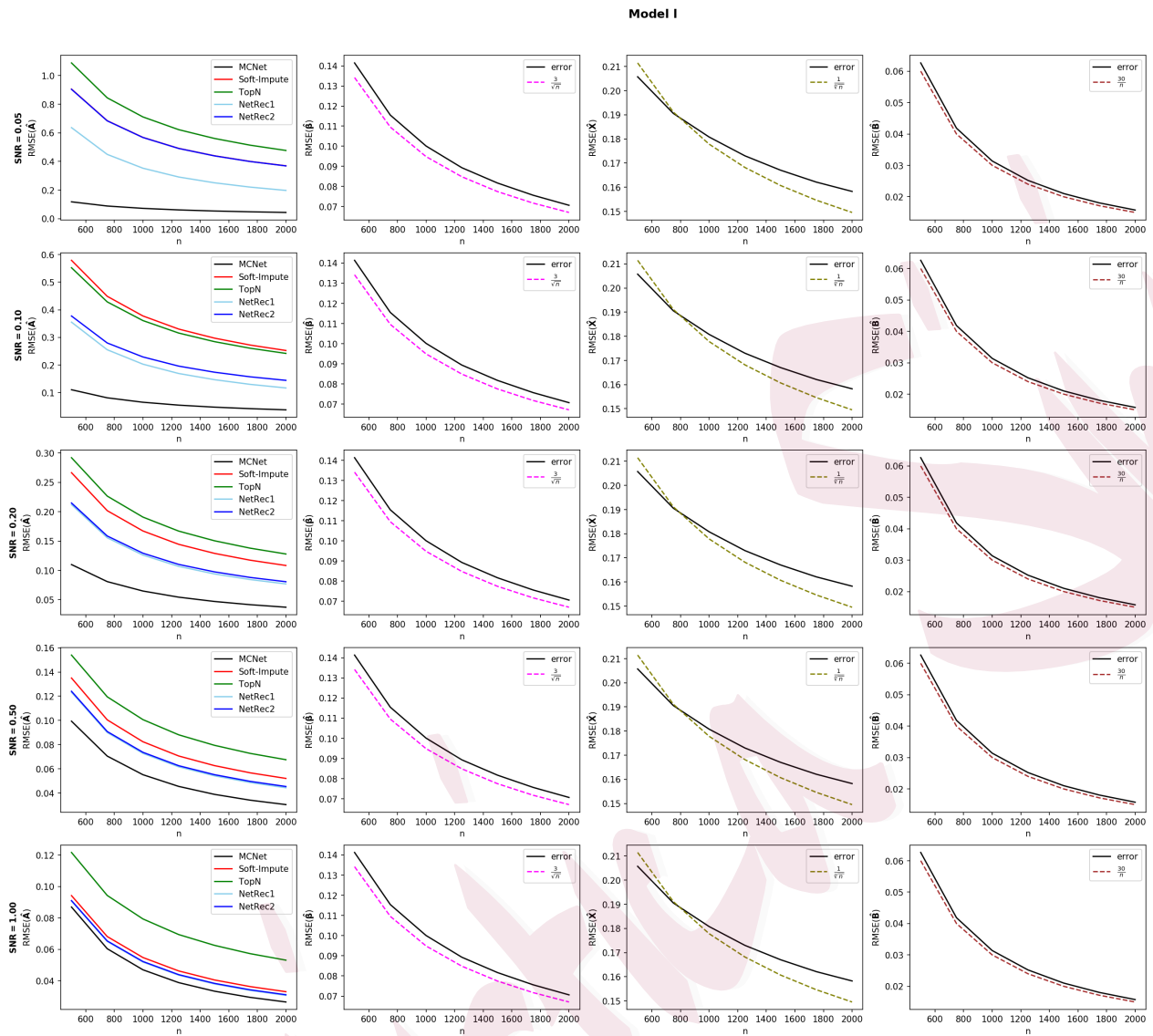

Figure 1: The estimation convergence under model I from 100 simulations.

Column 1 shows the comparisons of $d\left(\widehat{\mathbf{A}}, \mathbf{A}_{0}\right)$ among five comparative methods. Column 2-4 show the $d\left(\widehat{\boldsymbol{\beta}}, \mathbf{O}^{\mathrm{T}} \boldsymbol{\beta}_{0}\right), d\left(\widehat{\mathbf{X}} \mathbf{O}^{\mathrm{T}}, \mathbf{X}\right)$, and $d\left(\widehat{\mathbf{B}}, \mathbf{B}_{0}\right)$ respectively.

$\mathbf{A}_{0}+\boldsymbol{\epsilon}$, where $\boldsymbol{\epsilon}$ is the error matrix with entries from independent zero mean normal distribution, with standard deviations varying from 0.2 to 1.6 by stepwise 0.2. Figure 3 shows that MCNet outperforms SoftImpute, TopN, 
4.2 Performance as the noise level varies22

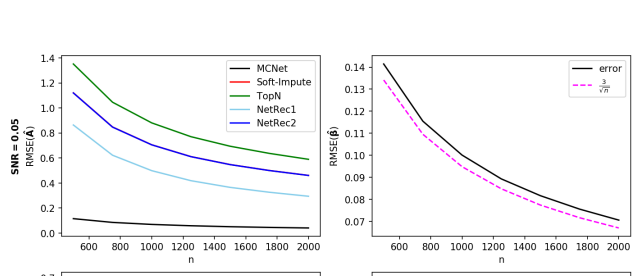

Model II
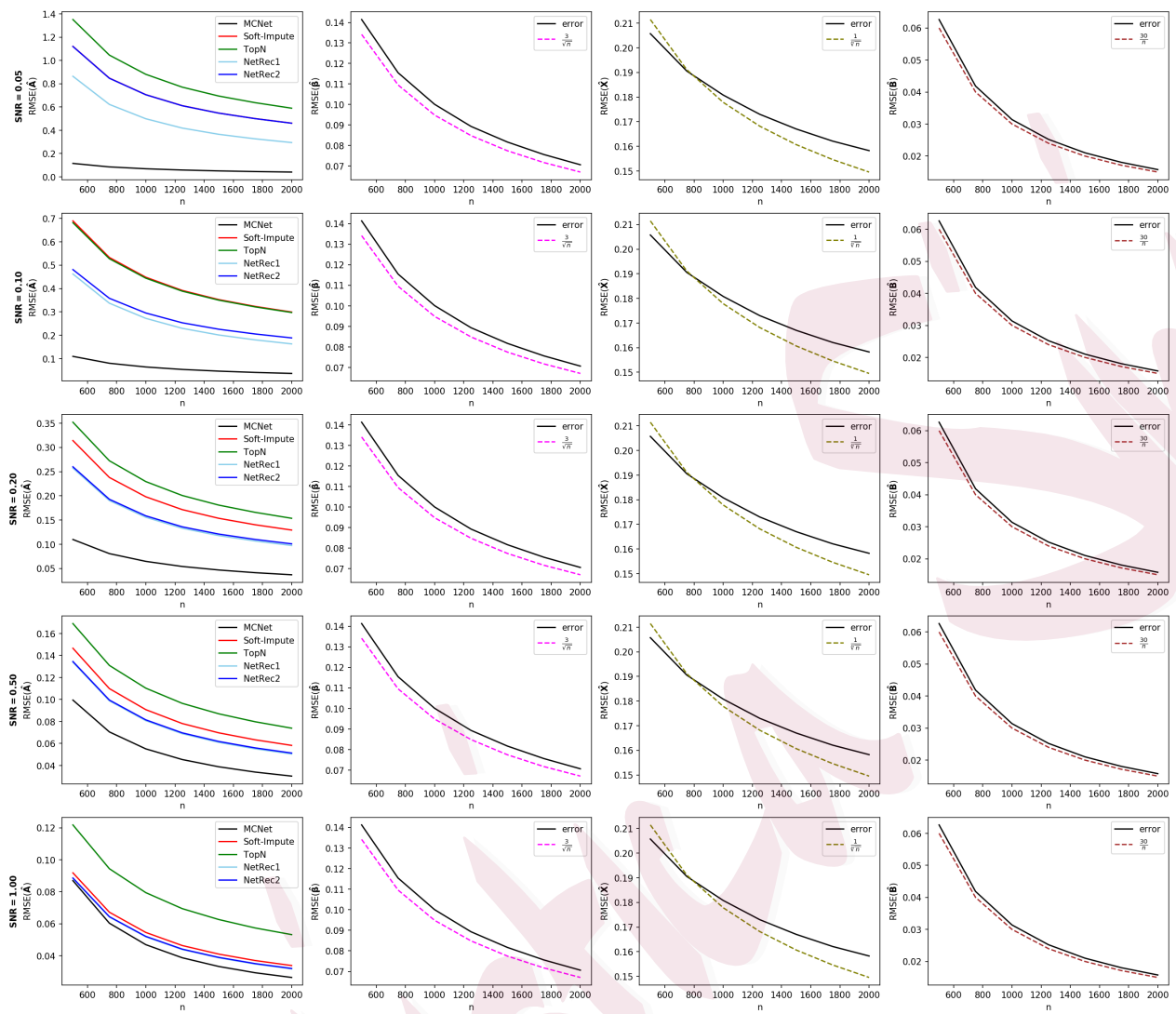

Figure 2: The estimation convergence under model II from 100 simulations. Column 1 shows the comparisons of $d\left(\widehat{\mathbf{A}}, \mathbf{A}_{0}\right)$ among five comparative methods. Column $2-4$ show the $d\left(\widehat{\boldsymbol{\beta}}, \mathbf{O}^{\mathrm{T}} \boldsymbol{\beta}_{0}\right), d\left(\widehat{\mathbf{X}} \mathbf{O}^{\mathrm{T}}, \mathbf{X}\right)$, and $d\left(\widehat{\mathbf{B}}, \mathbf{B}_{0}\right)$ respectively.

and NetRec with smaller $d\left(\widehat{\mathbf{A}}, \mathbf{A}_{0}\right)$. The results are consistent with our finding in Remark 1 of Section 3 that the performance of NetRec estimator in Jing et al. (2019) deteriorates while our estimator still has satisfactory 
4.3 Performance as the network density varies23

\begin{tabular}{|c|c|c|c|c|c|c|c|c|c|c|}
\hline & \multicolumn{5}{|c|}{ Model I } & \multicolumn{5}{|c|}{ Model II } \\
\hline & \multicolumn{5}{|c|}{$S N R$} & \multicolumn{5}{|c|}{$S N R$} \\
\hline & 0.05 & 0.1 & 0.2 & 0.5 & 1.0 & 0.05 & 0.1 & 0.2 & 0.5 & 1.0 \\
\hline \multirow[t]{2}{*}{ MCNet } & 2.70 & 2.44 & 2.43 & 2.09 & 1.80 & 2.59 & 2.41 & 2.43 & 2.09 & 1.80 \\
\hline & $(0.04)$ & $(0.03)$ & $(0.02)$ & $(0.02)$ & $(0.01)$ & $(0.03)$ & $(0.02)$ & $(0.02)$ & $(0.02)$ & $(0.01)$ \\
\hline \multirow[t]{2}{*}{ SoftImpute } & 21.36 & 14.14 & 6.28 & 3.10 & 2.08 & 26.53 & 16.79 & 7.44 & 3.41 & 2.06 \\
\hline & $(0.39)$ & $(0.17)$ & $(0.10)$ & $(0.04)$ & $(0.03)$ & $(0.44)$ & $(0.20)$ & $(0.11)$ & $(0.05)$ & $(0.03)$ \\
\hline \multirow[t]{2}{*}{ TopN } & 26.63 & 13.52 & 7.14 & 3.77 & 2.97 & 33.00 & 16.64 & 8.60 & 4.13 & 2.98 \\
\hline & $(0.32)$ & $(0.16)$ & $(0.08)$ & $(0.04)$ & $(0.04)$ & $(0.38)$ & $(0.19)$ & $(0.10)$ & $(0.05)$ & $(0.04)$ \\
\hline \multirow[t]{2}{*}{ NetRec1 } & 13.43 & 7.68 & 4.76 & 2.75 & 1.97 & 18.88 & 10.27 & 5.87 & 3.04 & 1.96 \\
\hline & $(0.30)$ & $(0.16)$ & $(0.09)$ & $(0.04)$ & $(0.03)$ & $(0.36)$ & $(0.19)$ & $(0.11)$ & $(0.04)$ & $(0.03)$ \\
\hline \multirow[t]{2}{*}{ NetRec2 } & 21.33 & 8.64 & 4.88 & 2.78 & 1.98 & 26.50 & 11.12 & 5.98 & 3.07 & 1.97 \\
\hline & $(0.39)$ & $(0.20)$ & $(0.10)$ & $(0.04)$ & $(0.03)$ & $(0.44)$ & $(0.22)$ & $(0.11)$ & $(0.05)$ & (0.03) \\
\hline
\end{tabular}

Table 1: Square root of the area under the $d^{2}(\widehat{\mathbf{A}}, \mathbf{A})$ curves with standard errors (in parentheses). The best results among the algorithms are indicated by the bold font.

result when the signal in $\mathbf{A}_{0}$ is small.

\subsection{Performance as the network density varies}

We compare MCNet, SoftImpute, TopN, and NetRec under the models with various network densities. We fix $d=10$ and $n_{1}=n_{2}=500$. To vary the density of the social network, we generate entries of $\mathbf{X}$ from a 
4.3 Performance as the network density varies24
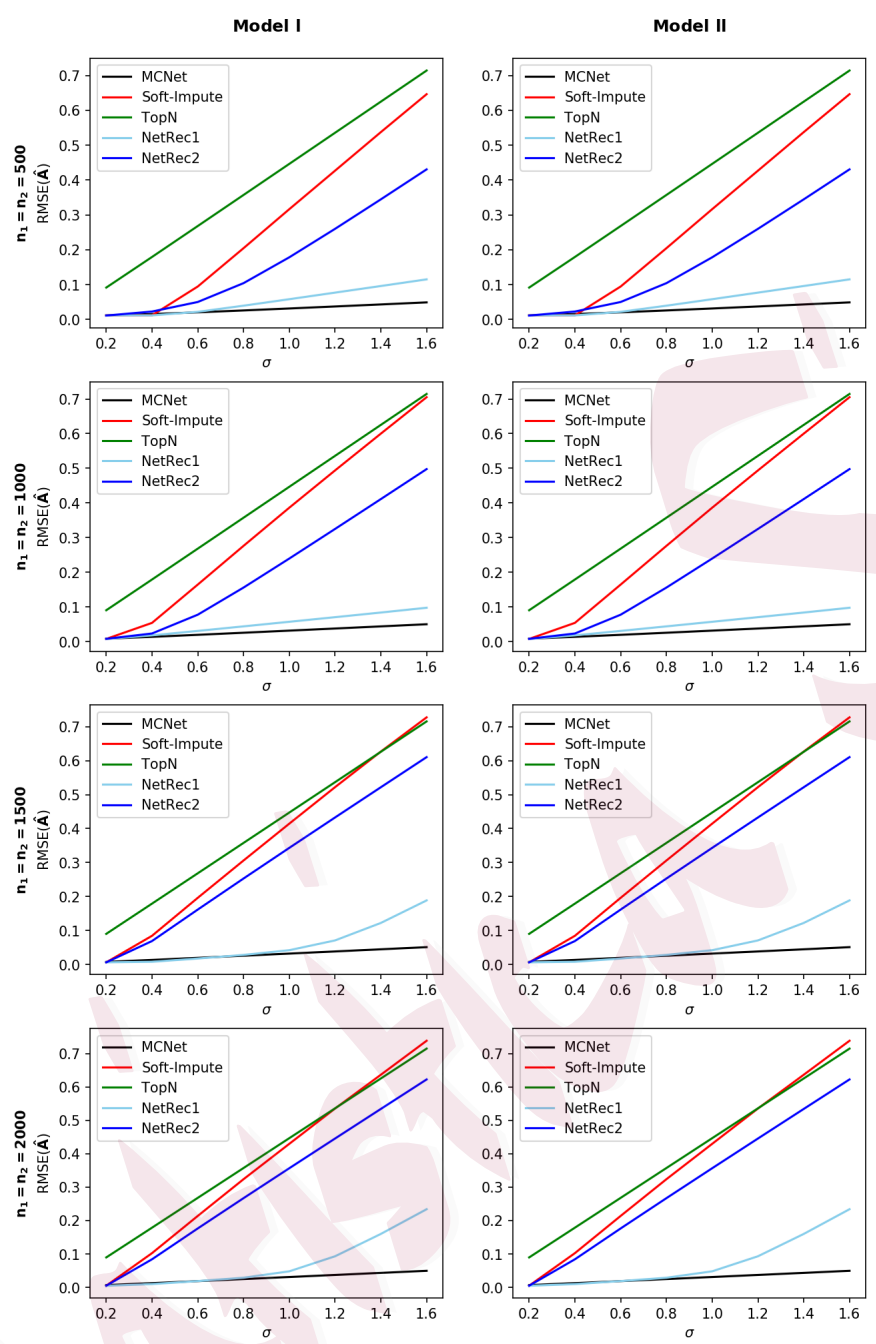

Figure 3: Performance of MCNet method and other methods under model

I and II for 100 repetitions.

beta distribution with parameters $(\alpha, 1)$, where $\alpha$ varies from 0.3 to 2.1 by stepwise 0.3 . We then scale the entries of $\mathbf{X}$ by a chosen constant to make sure $\max _{i, j} \mathbf{X}_{i} \cdot \mathbf{X}_{j}^{\mathrm{T}}=0.9$. The adjacency matrix is generated from a 


\subsection{Robustness of MCNet25}

Bernoulli distribution with success rate $\mathbf{X} \mathbf{X}^{\mathrm{T}}$. The parameters $\boldsymbol{\beta}_{0}$ and $\mathbf{B}_{0}$ are assumed to be the same as those in Section 4.1. Let $\mathbf{A}_{0}=c\left(\mathbf{X} \boldsymbol{\beta}_{0}+\mathbf{B}_{0}\right)$ where $c$ is a constant chosen to achieve $\left\|\mathbf{A}_{0}\right\|_{F}=1000$ and $\mathbf{Y}=\mathbf{A}_{0}+\boldsymbol{\epsilon}$ where $\boldsymbol{\epsilon}$ is an error matrix with independent mean zero normal entries. The standard deviation of the errors is chosen to achieve 0.5 signal-to-noise ratio. We only adopt model I for the missing mechanism. That is, each entry of the observation matrix $\mathbf{W}$ is generated from a Bernoulli distribution with mean 0.2 .

Figure 4 shows the resulting $d\left(\widehat{\mathbf{A}}, \mathbf{A}_{0}\right)$ with $95 \%$ confidence intervals versus the mean density of the social network (mean of $\boldsymbol{\Pi}=\mathbf{X X}^{\mathrm{T}}$ ). It indicates that our estimator is better than other comparative methods with smaller RMSEs on average. Furthermore, the advantages over other estimators are move obvious when the connective rates are higher.

\subsection{Robustness of MCNet}

We further evaluate MCNet when model (2.1) is violated. We select $n_{1}=$ 2000, $n_{2}=500$ and generate $\mathbf{Y}$ from $\mathbf{Y}=\mathbf{A}_{0}+\boldsymbol{\epsilon}, \mathbf{A}_{0}=\mathbf{U} \boldsymbol{\Sigma} \mathbf{V}^{\mathrm{T}} \operatorname{std}\left(\mathbf{U} \boldsymbol{\Sigma} \mathbf{V}^{\mathrm{T}}\right)^{-1}$ with $\mathbf{U} \in \mathbb{R}^{n_{1} \times 10}, \mathbf{V} \in \mathbb{R}^{n_{2} \times 10}$ being unitary matrices, $\boldsymbol{\Sigma}$ being the diagonal matrix with entries from the beta distribution with shape parameters 1,5 , and $\operatorname{std}\left(\mathbf{U} \boldsymbol{\Sigma} \mathbf{V}^{\mathrm{T}}\right)$ being the element-wise standard deviation of the matrix 


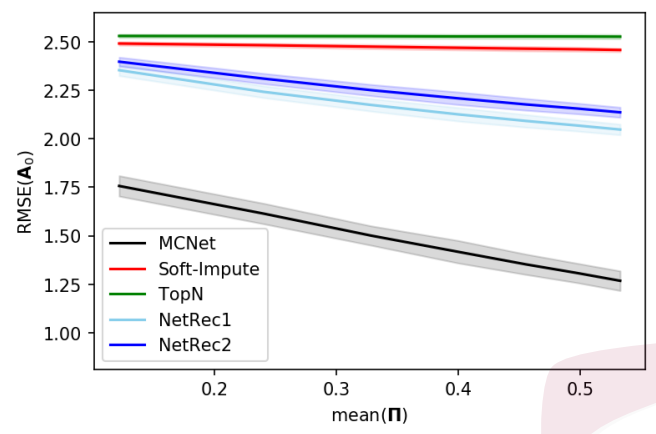

Figure 4: Connection probability. Performance of MCNet and other methods under model I for 100 repetitions.

$\mathbf{U} \mathbf{\Sigma} \mathbf{V}^{\mathrm{T}}$. The errors are generated from mean zero normal distribution, with standard deviations varying from 0.2 to 1.6. The entries are missing with probability 0.95. Furthermore, we generate $M_{i j}, M_{j i}$ from Bernoulli distribution with success rate to be the $i, j$ th entry of $\exp (-\beta \mathbf{T})$, where $\mathbf{T}$ is a matrix with $i, j$ th entry to be $\left\|\mathbf{A}_{0, i}-\mathbf{A}_{0, j} \cdot\right\|_{2}$ and $\beta$ is selected to allow the mean number of edges on each node of the network to be eight.

Figure 5 shows the resulting $d\left(\widehat{\mathbf{A}}, \mathbf{A}_{0}\right)$ from MCNet and NetRec with their $95 \%$ confidence intervals. The results show that when the true model deviates from the one assumed in MCNet, MCNet still provides satisfactory results, and it outperforms NetRec (with the correct model assumption) with smaller $d\left(\widehat{\mathbf{A}}, \mathbf{A}_{0}\right)$ when $\sigma_{i j}>2.5$. 


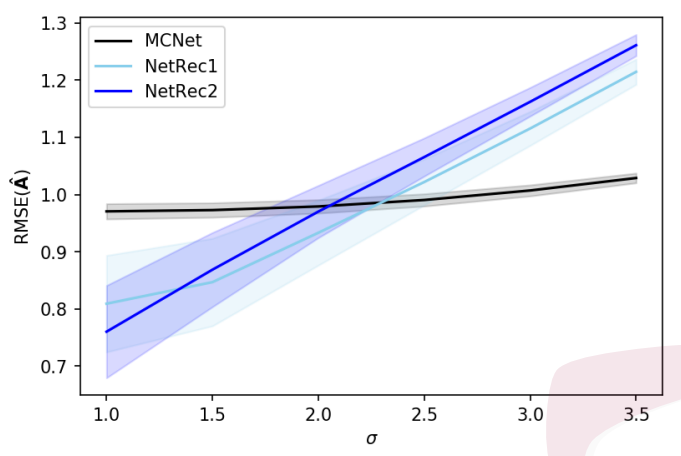

Figure 5: Robustness to assumptions. Performance of MCNet and NetRec methods under model I from 100 simulations.

\section{Empirical study}

In this section, we evaluate MCNet on two real datasets, including the Douban Movie Dataset from Douban.com and processed by Zheng et al. (2017), and the Yelp Dataset from the Yelp Dataset Challenge (Yelp (2019)).

\subsection{Douban Movie Data Analysis}

The dataset contains 195,493 ratings from 3,022 users on 6,971 movies. We select the a subset of movies that have at least ten ratings into the analysis. Furthermore, two users are considered as connected if they are friends. The $i, j$ th entry of $\mathbf{Y}$ is the rating from user $i$ on the $j$ th movie scaled by the total number of ratings received by the movie. Finally, the dataset comprises 3,022 users, 3,810 movies, and 176,656 ratings. And there 
5.1 Douban Movie Data Analysis28

are 1,366 connections in the social network. The sample standard deviation of observed entries of $\mathbf{Y}$ after scaling is 0.08 .

We select $d$ by using the Ahn and Horenstein's method (Ahn and Horenstein (2013)). We assume that the missing mechanism follows model I. We randomly split the users' ratings and the corresponding missing indicators into training (80\%) and testing (20\%) datasets. Within the training steps, we implement five-fold cross-validation to select the tuning parameters for MCNet, meanwhile we keep the social network connections fixed. We report the RMSE for the observed entries on the testing set and compare our method with SoftImpute, TopN, and NetRec. We repeat the trainingtesting procedure 50 times and report the testing RMSEs in Table 2 and Figure 6. The results show that MCNet has significantly smaller testing RMSE than the competing methods, where its $95 \%$ confidence interval does not overlap with the mean RMSEs from the other methods.

\begin{tabular}{cccccc}
\hline & MCNet & SoftImpute & TopN & NetRec1 & NetRec2 \\
\hline Mean of RMSEs & $\mathbf{0 . 0 1 1 8 7}$ & 0.01299 & 0.01258 & 0.01225 & 0.01251 \\
95\% CI & $0.01162-$ & $0.01273-$ & $0.01232-$ & $0.01190-$ & $0.01223-$ \\
& 0.01211 & 0.01324 & 0.01283 & 0.01260 & 0.01279 \\
\hline
\end{tabular}

Table 2: The mean out-of-sample RMSEs over 50 repetitions for Douban dataset. The minimum value is indicated by the bold font. 


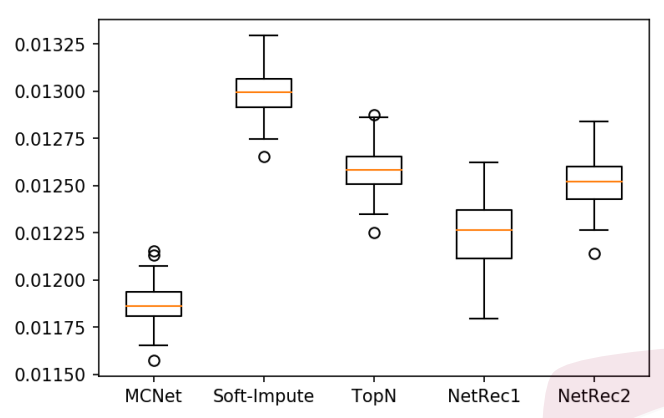

Figure 6: The testing RMSEs of MCNet and other methods over 50 repetitions.

\subsection{Yelp Data Analysis}

The dataset contains six million reviews from one million users on 192 thousand businesses. We extract the users' social network connections and their ratings on restaurants from five cities: Mentor OH, Gastonia NC, Matthews NC, Laval QC (Canada), and Brampton ON (Canada). Two users are connected if they are friends. The $i, j$ th entry of $\mathbf{Y}$ is the rating from user $i$ on the $j$ th restaurant scaled by the total number of reviews received by the restaurant. We provide some descriptive statistics for the five cities in Table 3 ,

We utilize Ahn and Horenstein's method (Ahn and Horenstein (2013)) to select $d$ and apply the same training-testing procedures in Section 5.1 to evaluate the methods. For each city, we repeat the training-testing pro- 
5.2 Yelp Data Analysis30

\begin{tabular}{lccccc}
\hline City & No. of users & No. of rstrnts. & No. of ratings & No. of conns. & Standard \\
& $\left(n_{1}\right)$ & $\left(n_{2}\right)$ & $\left(\sum_{i j} W_{i j}\right)$ & $\left(\sum_{i j} M_{i j}\right)$ & deviation \\
\hline Mentor & 2,611 & 181 & 4,689 & 2,334 & 0.18 \\
Gastonia & 3,050 & 184 & 5,000 & 3,422 & 0.15 \\
Matthews & 5,505 & 207 & 9,600 & 13,808 & 0.13 \\
Laval & 1,380 & 282 & 2,843 & 3,064 & 0.35 \\
Brampton & 4,329 & 546 & 9,283 & 12,392 & 0.25 \\
\hline
\end{tabular}

Table 3: Description of the data from five cities. Abbreviations: rstrnts, restaurants; conns, connections. Standard derivation is the sample standard deviation of observed entries of $\mathbf{Y}$ after scaling.

cedure 50 times. The results are reported in Table 4 and Figure 7, which show that all methods perform equivalently as the $95 \%$ confidence intervals overlap with each other, while MCNet has consistently smaller mean RMSE across all cities. We further calculate the sample standard deviation of the entries of $\mathbf{Y}$ in Table 3, which suggests the observed rating in Yelp has larger variation than that in Douban dataset (sample standard deviation is 0.08). This phenomenon suggests that when data variation is large, all the methods have equal performance, possibly due to the fact that the signal is weak and there is no much room for improvement. When the variation of the observed rating is small, our method performs significantly better than 
the other methods.

\begin{tabular}{|c|c|c|c|c|c|c|}
\hline & \multicolumn{2}{|c|}{ Mentor } & \multicolumn{2}{|c|}{ Gastonia } & \multicolumn{2}{|c|}{ Matthews } \\
\hline & Mean & $95 \% \mathrm{CI}$ & Mean & $95 \%$ CI & Mean & $95 \% \mathrm{CI}$ \\
\hline MCNet & 0.0479 & $0.0370-0.0588$ & 0.0334 & $0.0256-0.0411$ & 0.0202 & $0.0161-0.0243$ \\
\hline SoftImpute & 0.0507 & $0.0397-0.0617$ & 0.0354 & $0.0273-0.0434$ & 0.0217 & $0.0176-0.0258$ \\
\hline TopN & 0.0507 & $0.0397-0.0617$ & 0.0349 & $0.0269-0.0429$ & 0.0214 & $0.0173-0.0255$ \\
\hline NetRec1 & 0.0496 & $0.0388-0.0604$ & 0.0349 & $0.0269-0.0430$ & 0.0215 & $0.0175-0.0256$ \\
\hline \multirow[t]{3}{*}{ NetRec2 } & 0.0496 & $0.0388-0.0604$ & 0.0350 & $0.0269-0.0430$ & 0.0215 & $0.0175-0.0256$ \\
\hline & \multicolumn{2}{|c|}{ Laval } & \multicolumn{2}{|c|}{ Brampton } & & \\
\hline & Mean & $95 \% \mathrm{CI}$ & Mean & $95 \%$ CI & & \\
\hline MCNet & 0.2060 & $0.1729-0.2390$ & 0.0958 & $0.0831-0.1084$ & & \\
\hline SoftImpute & 0.2366 & $0.2033-0.2700$ & 0.1020 & $0.0897-0.1144$ & & \\
\hline TopN & 0.2351 & $0.2018-0.2684$ & 0.1004 & $0.0881-0.1128$ & & \\
\hline NetRec1 & 0.2235 & $0.1904-0.2566$ & 0.1005 & $0.0882-0.1128$ & & \\
\hline NetRec2 & 0.2213 & $0.1881-0.2545$ & 0.0996 & $0.0873-0.1119$ & & \\
\hline
\end{tabular}

Table 4: The mean testing RMSEs over 50 repetitions for the five cities. The minimum value of each column is indicated by the bold font.

\section{Conclusion}

We propose the MCNet method to incorporate social network information for matrix completion. MCNet generates latent features from the social network through the random dot product graph model, and the features 

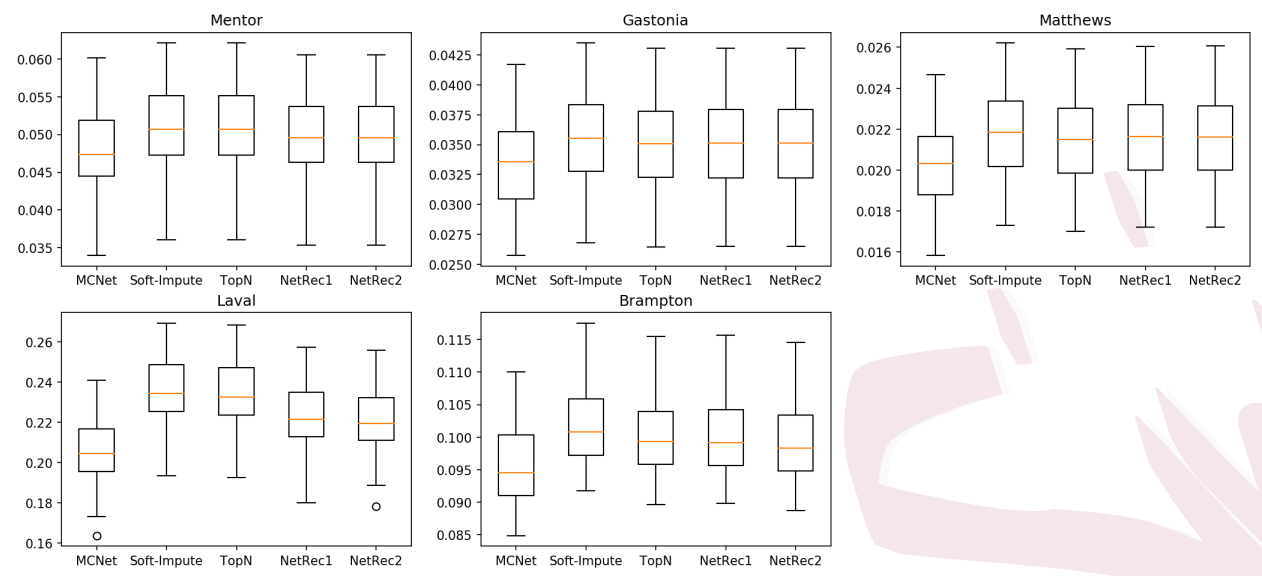

Figure 7: The testing RMSEs of MCNet and other methods over 50 repetitions.

are used in the matrix completion to reduce the estimation errors. The algorithm is evaluated through extensive simulations and two real data analyses from the Douban movie dataset and the Yelp dataset. The results show that MCNet outperforms the existing SoftImpute, TopN, and NecRec algorithms and provides robust performance when the signal-to-noise ratios are small.

Incorporating information in $\mathbf{Y}$ to estimate $\mathbf{X}$ will generally improve the estimation accuracy (Yu, Rao and Dhillon (2016)). To achieve this goal, we could add one more penalty term $\left\|\mathbf{M}-\mathbf{X X}^{\mathrm{T}}\right\|_{F}$ to the loss function in (2.4) and 2.5. This enriched loss function will allow us to obtain $\widehat{\mathbf{X}}, \widehat{\boldsymbol{\beta}}$ and $\widehat{\mathbf{B}}$ in a single framework. However, this penalty function is non-convex 
in $\mathbf{X}$, which brings difficulties in parameter estimation. Future work along this line is worthwhile to explore but out of the scope of the current paper.

\section{Supplementary Materials}

The supplementary material contains the detailed proofs of Lemma 3.1. 3.3 and Theorem 3.13 .2 .

\section{Acknowledgements}

The authors thank the reviewers and the associate editor for their constructive comments which led to a much improved version of the paper.

\section{References}

Abernethy, J., Bach, F., Evgeniou, T. and Vert, J.-P. (2009). A new approach to collaborative filtering: operator estimation with spectral regularization. Journal of Machine Learning Research 10, 803-826.

Ahn, S. C. and Horenstein, A. R. (2013). Eigenvalue ratio test for the number of factors. Econometrica 81, 1203-1227.

Athreya, A., Priebe, C. E., Tang, M., Lyzinski, V., Marchette, D. J. and Sussman, D. L. (2018). Statistical inference on random dot product graphs: a survey. The Journal of Machine Learning Research 18, 1-92.

Athreya, A., Priebe, C. E., Tang, M., Lyzinski, V., Marchette, D. J. and Sussman, D. L. (2016). 


\section{REFERENCES}

A limit theorem for scaled eigenvectors of random dot product graphs. Sankhya A 78, 1-18.

Bi, X., Qu, A., Wang, J. and Shen, X.(2017). A group-specific recommender system. Journal of the American Statistical Association 112, 1344-1353.

Cai, J. F., Candès, E. J. and Shen, Z. (2010). A singular value thresholding algorithm for matrix completion. SIAM Journal on optimization 20, 1956-1982.

Cai, T. T. and Zhou, W. X. (2018). Matrix completion via max-norm constrained optimization. Electronic Journal of Statistics 10, 1493-1525.

Candes, E. J. and Plan, Y. (2010). Matrix completion with noise. Proceedings of the IEEE 98, 925-936.

Chiang, K.-Y., Hsieh, C.-J. and Dhillon, I. S. (2015). Matrix completion with noisy side information. In Advances in Neural Information Processing Systems, 3447-3455.

Dai, B., Wang, J., Shen, X. and Qu, A. (2019). Smooth neighborhood recommender systems. Journal of machine learning research 20, 1-24.

Diallo, A. O., Diop, A. and Dupuy, J. F. (2017). Asymptotic properties of the maximumlikelihood estimator in zero-inflated binomial regression. Communications in StatisticsTheory and Methods 46, 9930-9948.

Elsener, A. and van de Geer, S. (2018). Robust low-rank matrix estimation. The Annals of Statistics 46, 3481-3509.

Fan, J., Gong, W. and Zhu, Z. (2019). Generalized high-dimensional trace regression via nuclear 


\section{REFERENCES}

norm regularization. Journal of Econometrics 212, 177-202.

Fan, J., Wang, W. and Zhu, Z. (2017). A shrinkage principle for heavy-tailed data: highdimensional robust low-rank matrix recovery. arXiv preprint arXiv:1603.08315.

Feuerverger, A., He, Y. and Khatri, S. (2012). Statistical significance of the Netflix challenge. Statistical Science 27, 202-231.

Foygel, R., Salakhutdinov, R., Shamir, O. and Srebro, N (2011). Learning with the weighted tracenorm under arbitrary sampling distributions. In Advances in Neural Information Processing Systems, 24.

Hall, D. B. (2000). Zero-inflated Poisson and binomial regression with random effects: a case study. Biometrics 56, 1030-1039.

Jing, B., Li, T., Ying, N. and Yu, X. (2019). Collaborative filtering with awareness of social network. Preprint.

Kang, Z., Peng, C. and Cheng, Q. (2016). Top-n recommender system via matrix completion. In Proceedings of the Thirtieth AAAI Conference on Artificial Intelligence, 179-185.

Kim, E., Lee, M. and Oh, S. (2015). Elastic-net regularization of singular values for robust subspace learning. In Proceedings of the IEEE conference on computer vision and pattern recognition, 915-923.

Klopp, O. (2014). Noisy low-rank matrix completion with general sampling distribution. Bernoulli 20, 282-303. 


\section{REFERENCES}

Koltchinskii, V., Lounici, K. and Tsybakov, A. B. (2011). Nuclear-norm penalization and optimal rates for noisy low-rank matrix completion. The Annals of Statistics 39, 2302-2329.

Lei, J. and Rinaldo, A. (2015). Consistency of spectral clustering in stochastic block models. The Annals of Statistics 43, 215-237.

Li, H., Chen, N. and Li, L. (2012). Error analysis for matrix elastic-net regularization algorithms. IEEE transactions on neural networks and learning systems $\mathbf{2 3}$, 737-748.

Liu, X. and Aberer, K. (2013). Soco: a social network aided context-aware recommender system. Proceedings of the 22nd international conference on World Wide Web, 781-802.

Liu, J. and Wu, C. (2017). Deep learning based recommendation: A survey. International Conference on Information Science and Applications, 451-458.

Lu, L. and Peng, X. (2013). Spectra of edge-independent random graphs. Electronic Journal of Combinatorics 20, 27.

Lyzinski, V., Sussman, D. L., Tang, M., Athreya, A. and Priebe, C. E. (2014) Perfect clustering for stochastic blockmodel graphs via adjacency spectral embedding. Electronic journal of statistics 8, 2905-2922.

Lyzinski, V., Tang, M., Athreya, A., Park, Y. and Priebe, C. E. (2016). Community detection and classification in hierarchical stochastic blockmodels. IEEE Transactions on Network Science and Engineering 4, 13-26.

Ma, H., Zhou, D., Liu, C., Lyu, M. R. and King, I. (2011). Recommender systems with social 


\section{REFERENCES}

regularization. In Proceedings of the fourth ACM international conference on Web search and data mining, 287-296.

Mao, X., Chen, S. X. and Wong, R. K. (2019). Matrix completion with covariate information. Journal of the American Statistical Association 114, 198-210.

Mao, X., Wong, R. K. and Chen, S. X. (2018). Matrix Completion under Low-Rank Missing Mechanism. arXiv preprint arXiv:1812.07813.

Mazumder, R., Hastie, T. and Tibshirani, R. (2010). Spectral regularization algorithms for learning large incomplete matrices. Journal of machine learning research 11, 2287-2322.

Negahban, S. and Wainwright, M. J. (2011). Estimation of (near) low-rank matrices with noise and high-dimensional scaling. The Annals of Statistics 39, 1069-1097.

Oliveira, R. I. (2009). Concentration of the adjacency matrix and of the laplacian in random graphs with independent edges. arXiv preprint arXiv:0911.0600.

Rao, N., Yu, H. F., Ravikumar, P. and Dhillon, I. S. (2015). Collaborative filtering with graph information: Consistency and scalable methods. In Advances in Neural Information Processing Systems, 7.

Recht, B. (2011). A Simpler Approach to Matrix Completion. Journal of Machine Learning Research 12, 3413-3430.

Recht, B., Fazel, M. and Parrilo, P. A. (2010). Guaranteed minimum-rank solutions of linear matrix equations via nuclear norm minimization. SIAM review 52, 471-501. 


\section{REFERENCES}

Robin, G., Wai, H.-T., Josse, J., Klopp, O. and Moulines, É. (2018). Low-rank interaction with sparse additive effects model for large data frames. In Advances in Neural Information Processing Systems, 5496-5506.

Rubin, D. B. (1976). Inference and missing data. Biometrika 63, 581-592.

Shi, Y., Larson, M. and Hanjalic, A. (2014). Collaborative filtering beyond the user-item matrix: a survey of the state of the art and future challenges. ACM Computing Surveys (CSUR) 47, $1-45$.

Srebro, N., Rennie, J. and Jaakkola, T. S. (2005). Maximum-margin matrix factorization. In Advances in Neural Information Processing Systems, 1329-1336.

Sun, T and Zhang, C. H. (2012). Calibrated elastic regularization in matrix completion. In Advances in Neural Information Processing Systems 863-871.

Sussman, D. L., Tang, M., Fishkind, D. E. and Priebe, C. E. (2012). A consistent adjacency spectral embedding for stochastic blockmodel graphs. Journal of the American Statistical Association 107, 1119-1128.

Sussman, D. L., Tang, M. and Priebe, C. E. (2013). Consistent latent position estimation and vertex classification for random dot product graphs. IEEE transactions on pattern analysis and machine intelligence 36, 48-57.

Tang, M., Athreya, A., Sussman, D. L., Lyzinski, V., Park, Y. and Priebe, C. E. (2017). A semiparametric two-sample hypothesis testing problem for random graphs. Journal of Computational and Graphical Statistics 26, 344-354. 


\section{REFERENCES}

Tang, M. and Priebe, C. E. (2018). Limit theorems for eigenvectors of the normalized laplacian for random graphs. The Annals of Statistics 46, 2360-2415.

Wang, H., Wang, N. and Yeung, D. Y. (2015). Collaborative deep learning for recommender systems. In Proceedings of the 21th ACM SIGKDD international conference on knowledge discovery and data mining, 1235-1244.

Xu, M., Jin, R. and Zhou, Z.-H. (2013). Speedup matrix completion with side information: application to multi-label learning. In Advances in Neural Information Processing Systems, 2301-2309.

Yelp (2019). Yelp dataset challenge @MISC. http://www.yelp.com/dataset_challenge.

Young, S. J. and Scheinerman, E. R. (2007). Random dot product graph models for social networks. In International Workshop on Algorithms and Models for the Web-Graph, 138149. Springer.

Yu, L., Pan, R. and Li, Z. (2011). Adaptive social similarities for recommender systems. In Proceedings of the fifth ACM conference on Recommender systems, 257-260.

Yu, H. F., Rao, N. and Dhillon, I. S. (2016). Temporal Regularized Matrix Factorization for High-dimensional Time Series Prediction. In Advances in Neural Information Processing Systems, 847-855.

Zhang, S., Yao, L., Sun, A. and Tay, Y. (2019). Deep learning based recommender system: A survey and new perspectives. ACM Computing Surveys (CSUR) 52, 1-38. 


\section{REFERENCES}

Zheng, J., Liu, J., Shi, C., Zhuang, F., Li, J. and Wu, B. (2017). Recommendation in heterogeneous information network via dual similarity regularization. International Journal of Data Science and Analytics 3, 35-48.

Zhu, Y., Shen, X. and Ye, C.(2016). Personalized prediction and sparsity pursuit in latent factor models. Journal of the American Statistical Association 111, 241-252.

Zou, H. and Hastie, T.(2005). Regularization and variable selection via the elastic net. Journal of the royal statistical society: series B (statistical methodology) 67, 301-320.

Department of Epidemiology \& Biostatistics, University of California, San Francisco, 550 16th St., San Francisco, CA 94158, USA.

E-mail: jingxuan.wang@ucsf.edu,fei.jiang@ucsf.edu

Innovation and Information Management, Faculty of Business and Economics, University of Hong Kong, Pokfulam Road, Hong Kong

E-mail: haipeng@hku.hk 